TRANSACTIONS OF THE

AMERICAN MATHEMATICAL SOCIETY

Volume 356, Number 7 , Pages $2927-2944$

S 0002-9947(03)03411-1

Article electronically published on November 18, 2003

\title{
MAPS BETWEEN NON-COMMUTATIVE SPACES
}

\author{
S. PAUL SMITH
}

\begin{abstract}
Let $J$ be a graded ideal in a not necessarily commutative graded $k$-algebra $A=A_{0} \oplus A_{1} \oplus \cdots$ in which $\operatorname{dim}_{k} A_{i}<\infty$ for all $i$. We show that the map $A \rightarrow A / J$ induces a closed immersion $i: \operatorname{Proj}_{n c} A / J \rightarrow \operatorname{Proj}_{n c} A$ between the non-commutative projective spaces with homogeneous coordinate rings $A$ and $A / J$. We also examine two other kinds of maps between non-commutative spaces. First, a homomorphism $\phi: A \rightarrow B$ between not necessarily commutative $\mathbb{N}$-graded rings induces an affine $\operatorname{map}^{\operatorname{Proj}_{n c} B} \supset U \rightarrow \operatorname{Proj}_{n c} A$ from a non-empty open subspace $U \subset \operatorname{Proj}_{n c} B$. Second, if $A$ is a right noetherian connected graded algebra (not necessarily generated in degree one), and $A^{(n)}$ is a Veronese subalgebra of $A$, there is a map $\operatorname{Proj}_{n c} A \rightarrow \operatorname{Proj}_{n c} A^{(n)}$; we identify open subspaces on which this map is an isomorphism. Applying these general results when $A$ is (a quotient of) a weighted polynomial ring produces a non-commutative resolution of (a closed subscheme of) a weighted projective space.
\end{abstract}

\section{INTRODUCTION}

This paper concerns maps between non-commutative projective spaces of the form $\operatorname{Proj}_{n c} A$. Before summarizing our main results we define the relevant terms.

Following Rosenberg [8, p. 278] and Van den Bergh [13], a non-commutative space $X$ is a Grothendieck category $\operatorname{Mod} X$. A map $g: Y \rightarrow X$ between two spaces is an adjoint pair of functors $\left(g^{*}, g_{*}\right)$ with $g_{*}: \operatorname{Mod} Y \rightarrow \operatorname{Mod} X$ and $g^{*}$ left adjoint to $g_{*}$. The map $g$ is affine [8, page 278] if $g_{*}$ is faithful and has a right adjoint. For example, a ring homomorphism $\varphi: R \rightarrow S$ induces an affine map $g: Y \rightarrow X$ between the affine spaces defined by $\operatorname{Mod} Y:=\operatorname{Mod} S$ and $\operatorname{Mod} X:=\operatorname{Mod} R$.

Let $k$ be a field. An $\mathbb{N}$-graded $k$-algebra $A$ is locally finite if $\operatorname{dim}_{k} A_{i}<\infty$ for all $i$. The non-commutative projective space $X$ with homogeneous coordinate ring $A$ is defined by

$$
\operatorname{Mod} X:=\operatorname{GrMod} A / \operatorname{Fdim} A
$$

(see Section 2), and

$$
\operatorname{Proj}_{n c} A:=\left(\operatorname{Mod} X, \mathcal{O}_{X}\right),
$$

where $\mathcal{O}_{X}$ is the image of $A$ in $\operatorname{Mod} X$. Thus $\operatorname{Proj}_{n c} A$ is an enriched quasi-scheme in the language of 13 . Let $Y$ be another non-commutative projective space with homogeneous coordinate ring $B$. A map $f: \operatorname{Proj}_{n c} B \rightarrow \operatorname{Proj}_{n c} A$ is a map $f: Y \rightarrow$ $X$ such that $f^{*} \mathcal{O}_{X} \cong \mathcal{O}_{Y}$.

Received by the editors September 18, 2002 and, in revised form, April 29, 2003.

2000 Mathematics Subject Classification. Primary 14A22; Secondary 16S38.

The author was supported by NSF grant DMS-0070560. 
When $A$ is a commutative $\mathbb{N}$-graded $k$-algebra we write $\operatorname{Proj} A$ for the usual projective scheme. We will always view a quasi-separated, quasi-compact scheme $X$ as a non-commutative space by associating to it the enriched space (Qcoh $\left.X, \mathcal{O}_{X}\right)$. The rule $X \mapsto\left(Q \operatorname{coh} X, \mathcal{O}_{X}\right)$ is a faithful functor.

Summary of results. The main results in this paper are Theorems 3.2, 3.3, 4.1 and Proposition 4.8.

A map $g: Y \rightarrow X$ is a closed immersion if it is affine and the essential image of $\operatorname{Mod} Y$ in $\operatorname{Mod} X$ under $g_{*}$ is closed under submodules and quotients. Theorem 3.2 shows that a surjective homomorphism $A \rightarrow A / J$ of graded rings induces a closed immersion $i: \operatorname{Proj}_{n c} A / J \rightarrow \operatorname{Proj}_{n c} A$. The functors $i^{*}$ and $i_{*}$ are the obvious ones (see the proof of 3.2). It seems to be a folklore result that $i^{*}$ is left adjoint to $i_{*}$, but we could not find a proof in the literature so we provide one here. Several people have been aware for some time that this is the appropriate intuitive picture, but, as far as I know, no formal definition of a closed immersion has been given and so no explicit proof has been given.

If $A$ is a graded subalgebra of $B$, commutative results suggest there should be a closed subspace $Z$ of $Y=\operatorname{Proj}_{n c} B$ and an affine map $g: Y \backslash Z \rightarrow \operatorname{Proj}_{n c} A$. Theorem 3.3 establishes such a result under reasonable hypotheses on $A$ and $B$. In fact, that result is set in a more general context, namely a homomorphism $\phi: A \rightarrow$ $B$ of graded rings. Corollary 3.4 then says that if $\phi: A \rightarrow B$ and $B$ is a finitely presented left $A$-module, then there is an affine map $g: \operatorname{Proj}_{n c} B \rightarrow \operatorname{Proj}_{n c} A$. This is a (special case of a) non-commutative analogue of the commutative result that a finite morphism is affine.

If $A$ is a quotient of a commutative polynomial ring, and $A^{(n)}$ is the graded subring with components $\left(A^{(n)}\right)_{i}=A_{n i}$, then there is an isomorphism of schemes $\operatorname{Proj} A \cong \operatorname{Proj} A^{(n)}$. Verevkin 12 proved that $\operatorname{Proj}_{n c} A \cong \operatorname{Proj}_{n c} A^{(n)}$ when $A$ is no longer commutative, but is connected and generated in degree one. Theorem 4.1 shows that when $A$ is not required to be generated in degree one, there is still a map $\operatorname{Proj}_{n c} A \rightarrow \operatorname{Proj}_{n c} A^{(n)}$, and Proposition 4.8 describes open subspaces on which this map is an isomorphism.

The results here are modelled on the commutative case, and none is a surprise. In large part the point of this paper is to make the appropriate definitions so that results from commutative algebraic geometry carry over verbatim to the noncommutative setting. Thus we formalize and make precise some of the terminology and intuition in papers like [2] and [7].

In Example 4.9 we show how our results apply to a quotient of a weighted polynomial ring to obtain a birational isomorphism $g: \operatorname{Proj}_{n c} A \rightarrow X=\operatorname{Proj} A$, where $X$ is a commutative subscheme of a weighted projective space. It can happen that $X$ is singular whereas $\operatorname{Proj}_{n c} A$ is smooth. Thus we can view $\operatorname{Proj}_{n c} A \rightarrow \operatorname{Proj} A$ as something like a non-commutative resolution of singularities. Furthermore, in this situation $g_{*} g^{*} \cong$ id.

We freely use basic notions and terminology for non-commutative spaces from the papers [9], [10], and [13].

\section{Definitions AND PRELIminaries}

Throughout this paper we assume that $A$ is a locally finite $\mathbb{N}$-graded algebra over a field $k$. Thus $A=A_{0} \oplus A_{1} \oplus \cdots$, and $\operatorname{dim}_{k} A_{i}<\infty$ for all $i$. The augmentation ideal $\mathfrak{m}$ of $A$ is $A_{1} \oplus A_{2} \oplus \cdots$. If $A_{0}$ is finite dimensional and $A$ is right noetherian, then it 
follows that $\operatorname{dim}_{k} A_{i}<\infty$ for all $i$ because $A_{\geq i} / A_{\geq i+1}$ is a noetherian $A / \mathfrak{m}$-module. We write $\operatorname{GrMod} A$ for the category of $\mathbb{Z}$-graded right $A$-modules, and define

Tails $A:=\operatorname{GrMod} A / \operatorname{Fdim} A$,

where $\operatorname{Fdim} A$ is the full subcategory consisting of direct limits of finite dimensional $A$-modules. Equivalently, Fdim $A$ consists of those modules in which every element is annihilated by a suitably large power of $\mathfrak{m}$. We write $\pi$ for the quotient functor $\operatorname{GrMod} A \rightarrow$ Tails $A$ and $\omega$ for its right adjoint.

The projective space with homogeneous coordinate ring $A$ is the space $X$ defined by $\operatorname{Mod} X:=$ Tails $A$. We write $\operatorname{Proj}_{n c} A=\left(\operatorname{Mod} X, \mathcal{O}_{X}\right)$, where $\mathcal{O}_{X}$ denotes the image of $A$ in Tails $A$.

A closed subspace $Z$ of a space $X$ is a full subcategory $\operatorname{Mod} Z$ of $\operatorname{Mod} X$ that is closed under submodules and quotient modules in $\operatorname{Mod} X$ and such that the inclusion functor $i_{*}: \operatorname{Mod} Z \rightarrow \operatorname{Mod} X$ has both a left adjoint $i^{*}$ and a right adjoint $i^{!}$.

Two spaces are isomorphic if their module categories are equivalent. Hence a map $Y \rightarrow X$ is a closed immersion if and only if it is an isomorphism from $Y$ to a closed subspace of $X$.

The complement $X \backslash Z$ to a closed subspace $Z$ is defined by

$$
\operatorname{Mod} X \backslash Z:=\operatorname{Mod} X / \mathrm{T}
$$

the quotient category of $\operatorname{Mod} X$ by the localizing subcategory $T$ consisting of those $X$-modules $M$ that are the direct limit of modules $N$ with the property that $N$ has a finite filtration $N=N_{n} \supset N_{n-1} \supset \cdots \supset N_{1} \supset N_{0}=0$ such that each $N_{i} / N_{i-1}$ is in $\operatorname{Mod} Z$. Because $T$ is a localizing category, there is an exact quotient functor $j^{*}: \operatorname{Mod} X \rightarrow \operatorname{Mod} X \backslash Z$, and its right adjoint $j_{*}: \operatorname{Mod} X \backslash Z \rightarrow \operatorname{Mod} X$. The pair $\left(j^{*}, j_{*}\right)$ defines a map $j: X \backslash Z \rightarrow X$. We call it an open immersion.

We sometimes write $\operatorname{Mod}_{Z} X$ for the category $T$ and call it the category of $X$ modules supported on $Z$.

Let $f: Y \rightarrow X$ be a map. If $f_{*}$ is faithful, then the counit id $Y \rightarrow f^{!} f_{*}$ is monic and the unit $f^{*} f_{*} \rightarrow \mathrm{id}_{Y}$ is epic.

Watt's Theorem for graded modules. Let $A$ and $B$ be $\mathbb{Z}$-graded $k$-algebras. We recall the analogue of Watt's Theorem proved by Del Rio [3 Proposition 3] that describes the $k$-linear functors $\operatorname{GrMod} A \rightarrow \operatorname{GrMod} B$ that have a right adjoint.

A bigraded $A$ - $B$-bimodule is an $A$ - $B$-bimodule

$$
M=\bigoplus_{(p, q) \in \mathbb{Z}^{2}}{ }^{2} M_{q}
$$

such that $A_{i \cdot p} M_{q} \cdot B_{j} \subset{ }_{i+p} M_{q+j}$ for all $i, j, p, q \in \mathbb{Z}$. Write $\otimes$ for $\otimes_{k}$. If $L$ is a graded right $A$-module, we define

$$
L \bar{\otimes}_{A} M:=\bigoplus_{q \in \mathbb{Z}}\left(L \bar{\otimes}_{A} M\right)_{q},
$$

where $\left(L \bar{\otimes}_{A} M\right)_{q}$ is the image of $\bigoplus_{p}\left(L_{-p} \otimes_{p} M_{q}\right)$ under the canonical map $L \otimes M \rightarrow$ $L \otimes_{A} M$. This gives $L \bar{\otimes}_{A} M$ the structure of a graded right $B$-module; it is a $B$ module direct summand of the usual tensor product $L \otimes_{A} M$.

If $N$ is a graded right $B$-module, we define

$$
\underline{\underline{\operatorname{Hom}}}_{B}(M, N):=\left\{f \in \operatorname{Hom}_{\mathrm{Gr} B}(M, N) \mid f\left({ }_{p} M_{*}\right)=0 \text { for almost all } p\right\} .
$$


This is made into a graded right $A$-module by declaring that $\operatorname{deg} f=p$ if $f\left({ }_{i} M_{*}\right)=0$ for all $i \neq-p$. Hence $\underline{\underline{\operatorname{Hom}}}_{B}(M, N)_{p}$ is naturally isomorphic to $\operatorname{Hom}_{\operatorname{Gr} B}\left(-p M_{*}, N\right)$, and there is a natural isomorphism

$$
\underline{\underline{\operatorname{Hom}}}_{B}(M, N)=\bigoplus_{p} \operatorname{Hom}_{\mathrm{Gr} B}\left({ }_{-p} M_{*}, N\right) .
$$

The usual adjoint isomorphism between Hom and $\otimes$ then induces an isomorphism

$$
\operatorname{Hom}_{\mathrm{Gr} B}\left(L \bar{\otimes}_{A} M, N\right) \cong \operatorname{Hom}_{\mathrm{Gr} A}\left(L, \underline{\underline{\operatorname{Hom}}}_{B}(M, N)\right),
$$

showing that $-\bar{\otimes}_{A} M: \operatorname{GrMod} A \rightarrow \operatorname{GrMod} B$ is left adjoint to $\underline{\underline{\operatorname{Hom}}}_{B}(M,-)$.

Theorem 2.1 (Del Rio 3). Let $A$ and $B$ be graded $k$-algebras, and $F: \operatorname{GrMod} A \rightarrow$ $\operatorname{GrMod} B$ a k-linear functor having a right adjoint. Then $F \cong-\bar{\otimes}_{A} M$, where $M$ is the bigraded $A$-B-bimodule

$$
M=\bigoplus_{p \in \mathbb{Z}} F(A(p))
$$

with homogeneous components ${ }_{p} M_{q}=F(A(p))_{q}$.

If $F$ also commutes with the twists by degree, then $F$ is given by tensoring with a graded $A$-B-bimodule, say $V=\bigoplus_{n} V_{n}$. The corresponding $M$ in this case is $M=\bigoplus V(p)$ with ${ }_{p} M_{q}=V(p)_{q}$.

The left $A$-action on $M$ is given by declaring that $x \in A_{i}$ acts on ${ }_{p} M_{*}$ as $F\left(\lambda_{x}\right)$, where $\lambda_{x}: A(p) \rightarrow A(p+i)$ denotes left multiplication by $x$.

\section{MAPS INDUCED BY GRADED RING HOMOMORPHISMS}

Throughout this section we assume that $A$ and $B$ are locally finite $\mathbb{N}$-graded algebras over a field $k$.

We consider the problem of when a homomorphism $\phi: A \rightarrow B$ of graded rings induces a map $g: \operatorname{Proj}_{n c} B \rightarrow \operatorname{Proj}_{n c} A$ and, if it does, how the properties of $g$ are determined by the properties of $\phi$.

Associated to $\phi$ is an adjoint triple $\left(f^{*}, f_{*}, f^{!}\right)$of functors between the categories of graded modules. Explicitly, $f^{*}=-\otimes_{A} B, f_{*}=-\otimes_{B} B_{A}$ is the restriction map, and $f^{!}=\bigoplus_{p \in \mathbb{Z}} \operatorname{Hom}_{\mathrm{Gr} B}(B(-p),-)$. We wish to establish conditions on $\phi$ which imply that these functors factor through the quotient categories in the following diagrams:

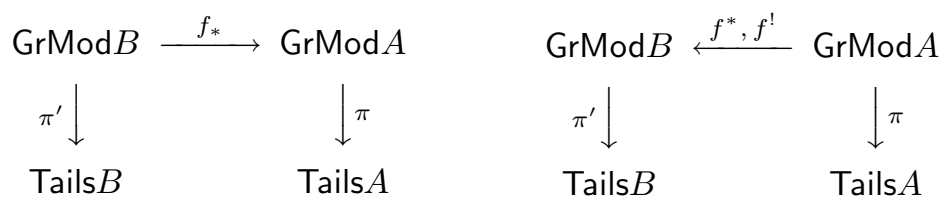

Lemma 3.1. Let $\mathrm{A}$ and $\mathrm{B}$ be Grothendieck categories with localizing subcategories $\mathrm{S} \subset \mathrm{A}$ and $\mathrm{T} \subset \mathrm{B}$. Let $\pi: \mathrm{A} \rightarrow \mathrm{A} / \mathrm{S}$ and $\pi^{\prime}: \mathrm{B} \rightarrow \mathrm{B} / \mathrm{T}$ be the quotient functors, and let $\omega$ and $\omega^{\prime}$ be their right adjoints. Consider the following diagram of functors:

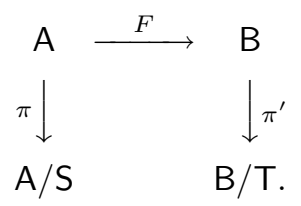


Suppose that $F(\mathrm{~S}) \subset \mathrm{T}$.

(1) There is a unique functor $G: \mathrm{A} / \mathrm{S} \rightarrow \mathrm{B} / \mathrm{T}$ such that $\pi^{\prime} F=G \pi$.

(2) If $H: \mathrm{B} \rightarrow \mathrm{A}$ is a right adjoint to $F$, then $\pi H \omega^{\prime}$ is a right adjoint to $G$.

(3) If $H$ is a right adjoint to $F$ and $G^{\prime}$ is a right adjoint to $G$, then $H(\mathrm{~T}) \subset \mathrm{S}$ if and only if $G^{\prime} \pi^{\prime} \cong \pi H$.

Proof. (1) The existence and uniqueness of $G$ is due to Gabriel [6, Corollaire 2, p. $368]$.

(2) To show that $G$ has a right adjoint it suffices to show that it is right exact and commutes with direct sums. If $\mathcal{M}_{\lambda}$ is a collection of objects in $\mathrm{A} / \mathrm{S}$, then each is of the form $\mathcal{M}_{\lambda}=\pi M_{\lambda}$ for some object $M_{\lambda}$ in A. Both $\pi^{\prime}$ and $F$ commute with direct sums because they have right adjoints, so $G \pi$ commutes with direct sums; $\pi$ also commutes with direct sums. Therefore

$$
G\left(\bigoplus \mathcal{M}_{\lambda}\right)=G\left(\bigoplus \pi M_{\lambda}\right) \cong G \pi\left(\bigoplus M_{\lambda}\right) \cong \bigoplus G \pi M_{\lambda}=\bigoplus G \mathcal{M}_{\lambda}
$$

Thus $G$ commutes with direct sums.

To see that $G$ is right exact, consider an exact sequence

$$
0 \rightarrow \mathcal{L} \rightarrow \mathcal{M} \rightarrow \mathcal{N} \rightarrow 0
$$

in A/S. By Gabriel 6, Corollaire 1, p. 368], (3-1) is obtained by applying $\pi$ to an exact sequence $0 \rightarrow L \rightarrow M \rightarrow N \rightarrow 0$ in A. Both $\pi^{\prime}$ and $F$ are right exact because they have right adjoints, so $\pi^{\prime} F L \rightarrow \pi^{\prime} F M \rightarrow \pi^{\prime} F N \rightarrow 0$ is exact. In other words, $G \mathcal{L} \rightarrow G \mathcal{M} \rightarrow G \mathcal{N} \rightarrow 0$ is exact.

Hence $G$ has a right adjoint, say $G^{\prime}$. It follows that $\omega G^{\prime}$ is a right adjoint to $G \pi$. But $G \pi=\pi^{\prime} F$ has $H \omega^{\prime}$ as a right adjoint, so $\omega G^{\prime} \cong H \omega^{\prime}$. Since $\pi \omega \cong \mathrm{id}_{\mathrm{A} / \mathrm{s}}$, $G^{\prime} \cong \pi H \omega^{\prime}$. Since a right adjoint is only determined up to natural equivalence, $\pi H \omega^{\prime}$ is a right adjoint to $G$.

(3) If $H(\mathbf{T}) \subset \mathrm{S}$, then $\pi H$ vanishes on $\mathbf{T}$ so, by Gabriel [6, Corollaire 2, page 368], there is a functor $V: \mathrm{B} / \mathrm{T} \rightarrow \mathrm{A} / \mathrm{S}$ such that $V \pi^{\prime}=\pi H$. Thus $V \cong \pi H \omega^{\prime}$, and this is isomorphic to $G^{\prime}$ by (2). Hence $G^{\prime} \pi^{\prime} \cong \pi H$. Conversely, if $G^{\prime} \pi^{\prime} \cong \pi H$, then $\pi H(\mathrm{~T})=0$, so $H(\mathrm{~T}) \subset \mathrm{S}$.

Warning. The functor $H$ in part (2) of Lemma 3.1 need not have the property that $H(\mathrm{~T})$ is contained in $\mathrm{S}$. An explicit example of this is provided by taking $\mathrm{B}=\mathrm{A}, F=H=\mathrm{id}_{\mathrm{A}}, \mathrm{S}=0$, and $\mathrm{T}=\mathrm{B}$.

Theorem 3.2. Let $J$ be a graded ideal in an $\mathbb{N}$-graded $k$-algebra $A$. Then the homomorphism $A \rightarrow A / J$ induces a closed immersion $i: \operatorname{Proj}_{n c} A / J \rightarrow \operatorname{Proj}_{n c} A$.

Proof. Write $X=\operatorname{Proj}_{n c} A$ and $Z=\operatorname{Proj}_{n c} A / J$. Write $\mathfrak{m}=A_{1} \oplus A_{2} \oplus \cdots$. Thus $\operatorname{Mod} X=\operatorname{GrMod} A / \operatorname{Fdim} A$. We write $\pi: \operatorname{GrMod} A \rightarrow \operatorname{Mod} X$ for the quotient functor and $\omega$ for a right adjoint to it. Similarly, $\pi^{\prime}: \operatorname{GrMod} A / J \rightarrow \operatorname{Mod} Z$ is the quotient functor, and $\omega^{\prime}$ is a right adjoint to it. See [12] and [1 Section 2] for more information about this.

Let $f_{*}: \operatorname{GrMod} A / J \rightarrow \operatorname{GrMod} A$ be the inclusion functor. It has a left adjoint $f^{*}=-\otimes_{A} A / J$, and a right adjoint $f^{!}$that sends a graded $A$-module to the largest submodule of it that is annihilated by $J$. 
By [6. Corollaire 2, p. 368] (= part (1) of Lemma 3.1), there is a unique functor $i_{*}$ such that

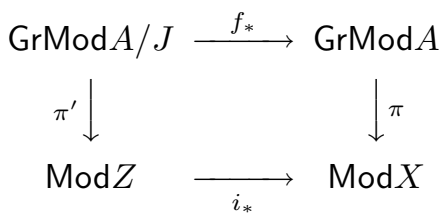

commutes, and $i_{*}$ is exact because $f_{*}$ is [6, Corollaire 3, p. 369]. Thus $i_{*} \pi^{\prime}=\pi f_{*}$.

By Lemma 3.1 $(2), i_{*}$ has a right adjoint, namely $i^{!}:=\pi^{\prime} f^{!} \omega$. It is clear that $f^{!}$ sends $\mathrm{F} \operatorname{dim} A$ to $\operatorname{Fdim} A / J$, so $\pi^{\prime} f^{!} \cong i^{!} \pi$ by Lemma 3.1(3).

It is clear that $f^{*}$ sends $\operatorname{Fdim} A$ to $\operatorname{Fdim} A / J$, so by [6, Corollaire 2, p. 368], there is a functor $i^{*}: \operatorname{Mod} X \rightarrow \operatorname{Mod} Z$ such that $\pi^{\prime} f^{*}=i^{*} \pi$. Since $f_{*}$ is right adjoint to $f^{*}$, it follows from Lemma 3.1 (2) that $\pi f_{*} \omega^{\prime}$ is a right adjoint to $i^{*}$. But $\pi f_{*} \omega^{\prime}=i_{*} \pi^{\prime} \omega^{\prime} \cong i_{*}$. Hence $i^{*}$ is left adjoint to $i_{*}$.

We now show that $i_{*}$ is faithful. Since $i_{*}$ has a left and a right adjoint, it is exact, so it suffices to show that if $i_{*} \mathcal{M}=0$, then $\mathcal{M}=0$. Suppose that $i_{*} \pi^{\prime} M=0$ for some $M \in \operatorname{GrMod} A / J$. Then $\pi f_{*} M=0$, and we conclude that $M$ is in $\operatorname{Fdim} A$, and hence in $\operatorname{Fdim} A / J$; therefore $\pi^{\prime} M=0$. Hence $i_{*}$ is faithful.

We will show that $i_{*}$ is full after establishing the following fact.

Claim. $\omega \pi f_{*} \cong f_{*} \omega^{\prime} \pi^{\prime}$.

Proof. Let $M \in \operatorname{GrMod} A / J$, let $\tau M$ denote the largest submodule of $M$ that is in $\operatorname{Fdim} A / J$ (equivalently, in $\operatorname{Fdim} A$ ), and set $\bar{M}=M / \tau M$. Then $\pi^{\prime} M=\pi^{\prime} \bar{M}$ and $\pi f_{*} M=\pi f_{*} \bar{M}$, so the two functors take the same value on $M$ if and only if they take the same value on $\bar{M}$. Hence we can, and will, assume that $M=\bar{M}$; i.e., $\tau M=0$.

We must show that $\omega \pi M=\omega^{\prime} \pi^{\prime} M$. By definition $\omega \pi M$ is the largest essential extension $0 \rightarrow M \rightarrow \omega \pi M \rightarrow T \rightarrow 0$ such that $T \in \operatorname{Fdim} A$. The definition of $\omega^{\prime} \pi^{\prime} M$ is analogous, although $T$ is now required to belong to $\mathrm{F} \operatorname{dim} A / J$. It therefore suffices to prove that $\omega \pi M$ is in $\operatorname{GrMod} A / J$. The top row in the diagram

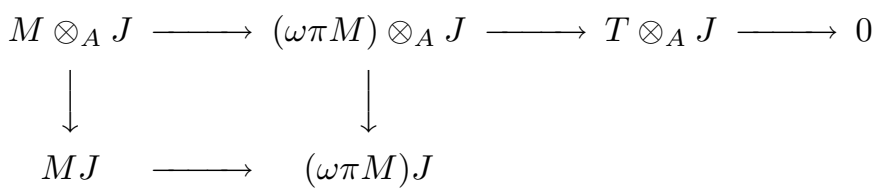

is exact, and the first vertical map is zero because $M$ is an $A / J$-module, so there is an induced map $T \otimes_{A} J \rightarrow(\omega \pi M) J$. This map is surjective. However, $T \otimes_{A}$ $J$ belongs to $\mathrm{F} \operatorname{dim} A$ because $T$ does, so $(\omega \pi M) J \in \mathrm{F} \operatorname{dim} A$. This implies that $M \cap(\omega \pi M) J \in \operatorname{Fdim} A$. But $\tau M=0$, so $M \cap(\omega \pi M) J=0$, and it follows that $(\omega \pi M) J=0$ because $M$ is essential in $\omega \pi M$. In other words, $\omega \pi M \in \operatorname{GrMod} A / J$. This completes the proof of the claim.

We have $f^{*} f_{*} \cong \mathrm{id}_{\operatorname{GrMod} A / J}$ and $\pi^{\prime} \omega^{\prime} \cong \mathrm{id}_{\operatorname{Mod} Z}$, so

$$
i^{*} i_{*} \cong\left(\pi^{\prime} f^{*} \omega\right)\left(\pi f_{*} \omega^{\prime}\right) \cong \pi^{\prime} f^{*} f_{*} \omega^{\prime} \pi^{\prime} \omega^{\prime} \cong \operatorname{id}_{\operatorname{Mod} Z} .
$$

It follows from this that $i_{*}$ is full.

To see that $i_{*}$ is a closed immersion, it remains to check that $i_{*}(\operatorname{Mod} Z)$ is closed under submodules and quotients in $\operatorname{Mod} X$. Let $\mathcal{M} \in \operatorname{Mod} Z$ and suppose that $0 \rightarrow \mathcal{L} \rightarrow i_{*} \mathcal{M} \rightarrow \mathcal{N} \rightarrow 0$ is an exact sequence in $\operatorname{Mod} X$. There is an exact sequence 
$0 \rightarrow \omega \mathcal{L} \rightarrow \omega i_{*} \mathcal{M} \rightarrow \omega \mathcal{N} \rightarrow R^{1} \omega \mathcal{L}$ in $\operatorname{GrMod} A$. Let $N$ denote the image of $\omega i_{*} \mathcal{M}$ in $\omega \mathcal{N}$. Then $\mathcal{L} \cong \pi \omega \mathcal{L}$ and $\mathcal{N} \cong \pi N$, because $\pi$ is exact and $R^{1} \omega \mathcal{L} \in \operatorname{Fdim} A$. Now $\mathcal{M}=\pi^{\prime} M$ for some $M \in \operatorname{GrMod} A / J$, so $\omega i_{*} \mathcal{M}=\omega i_{*} \pi^{\prime} M=\omega \pi f_{*} M \cong$ $f_{*} \omega^{\prime} \pi^{\prime} M$, from which we conclude that $\omega i_{*} \mathcal{M}$ is annihilated by $J$. Therefore $\omega \mathcal{L}$ is also annihilated by $J$, so is of the form $f_{*} L$ for some $L \in \operatorname{GrMod} A / J$; hence $\mathcal{L} \cong \pi \omega \mathcal{L} \cong \pi f_{*} L \cong i_{*} \pi^{\prime} L \in i_{*}(\operatorname{Mod} Z)$. Since $N$ is a quotient of $\omega i_{*} \mathcal{M}$ it is also annihilated by $J$, and a similar $\operatorname{argument}$ shows that $\mathcal{N} \in i_{*}(\operatorname{Mod} Z)$.

Since $f^{*} A=A / J, i^{*} \mathcal{O}_{X}=\mathcal{O}_{Z}$.

We retain the notation of the theorem.

Because $i_{*}$ is fully faithful, we often view $\operatorname{Mod} Z$ as a full subcategory of $\operatorname{Mod} X$ and speak of $Z=\operatorname{Proj}_{n c} A / J$ as a closed subspace of $X=\operatorname{Proj}_{n c} A$ and call it the zero locus of $J$.

It is not the case that every closed subspace of $\operatorname{Proj}_{n c} A$ is the zero locus of a two-sided ideal in $A$. For example, if $A=k_{q}[x, y]$ is the ring defined by the relation $y x=q x y$ where $0 \neq q \in k$, then $\operatorname{Proj}_{n c} A \cong \mathbb{P}^{1}$, but the closed points of $\mathbb{P}^{1}$ are not cut out by two-sided ideals when $q \neq 1$ : for example, $(\alpha x+\beta y) A$ is not a two-sided ideal when $q \neq 1$ and $\alpha \beta \neq 0$. This is essentially due to the fact that the auto-equivalence $\mathcal{M} \rightarrow \mathcal{M}(1)$ of $\operatorname{Mod} X$ induced by the degree shift on $A$ does not generally send $Z$-modules to $Z$-modules.

A more difficult question is whether every closed subspace of $\operatorname{Proj}_{n c} A$ is the zero locus of a two-sided ideal in some homogeneous coordinate ring of $\operatorname{Proj}_{n c} A$. We do not know the answer to this question.

Theorem 3.3. Suppose that $\phi: A \rightarrow B$ is a map of locally finite $\mathbb{N}$-graded $k$ algebras. Write $X=\operatorname{Proj}_{n c} A$ and $Y=\operatorname{Proj}_{n c} B$. Let $\mathfrak{m}$ be the augmentation ideal of $A$, and let $I$ be the largest two-sided ideal of $B$ contained in $\phi(\mathfrak{m}) B$. Let $Z \subset Y$ be the zero locus of $I$. If $B \phi(\mathfrak{m})^{n} \subset \phi(\mathfrak{m}) B$ for some integer $n$, then $\phi$ induces an affine map

$$
g: Y \backslash Z \rightarrow X .
$$

Proof. The category of modules over $Y \backslash Z$ is $\operatorname{Mod} Y / \operatorname{Mod}_{Z} Y$. This is equivalent to the quotient category $\operatorname{GrMod} B / \mathrm{T}$, where $\mathrm{T}$ consists of those modules $M$ with the property that every element of $M$ is killed by some power of $I$. Let $\pi^{\prime}: \operatorname{GrMod} B \rightarrow$ $\operatorname{GrMod} B / \mathrm{T}$ be the quotient functor. We have functors $\left(f^{*}, f_{*}, f^{!}\right)$between the graded module categories and a diagram

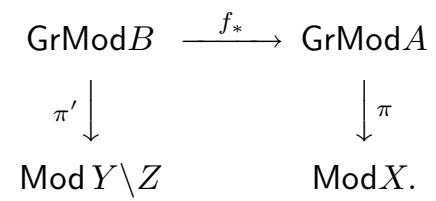

To check that $f^{*}$ sends $\operatorname{Fdim} A$ to $\mathbf{T}$, it suffices to check that $f^{*}(A / \mathfrak{m}) \in \mathbf{T}$, because $f^{*}$ commutes with direct limits and with the degree twist (1). However, $f^{*}(A / \mathfrak{m})=B / \phi(\mathfrak{m}) B$ is in $\mathrm{T}$ because $I \subset \phi(\mathfrak{m}) B$. Hence there is a unique functor $g^{*}:$ Tails $A \rightarrow(\operatorname{GrMod} B) / \mathrm{T}$ satisfying $g^{*} \pi=\pi^{\prime} f^{*}$.

To check that $f_{*}$ sends $\mathrm{T}$ to $\mathrm{F} \operatorname{dim} A$, it suffices to check that $f_{*}(B / I)$ is in $\mathrm{Fdim} A$. However, $(B / I) \mathfrak{m}^{n}=B \phi(\mathfrak{m})^{n}+I / I$; the hypothesis that $B \phi(\mathfrak{m})^{n} \subset \phi(\mathfrak{m}) B$ ensures that $B \phi(\mathfrak{m})^{n} \subset I$, so $(B / I) \mathfrak{m}^{n}=0$. Hence there is an exact functor $g_{*}: \operatorname{GrMod} B / \mathrm{T} \rightarrow$ Tails $A$ such that $g_{*} \pi^{\prime}=\pi f_{*}$. 
By Lemma 3.1 (2), $g_{*}$ has a right adjoint $g^{!}=\pi^{\prime} f^{!} \omega$.

To show that $g_{*}$ is faithful we must show that if $M$ is a graded $B$-module such that $g_{*} \pi^{\prime} M=0$, then $M \in \mathrm{T}$. Since $\pi f_{*} M=0$, as an $A$-module $M$ is a direct limit $\stackrel{\lim }{\longrightarrow} M_{\lambda}$ where each $M_{\lambda}$ is a finite dimensional $A$-module. There is an epimorphism

$$
\underset{\lim _{(}}{\longrightarrow}\left(M_{\lambda} \otimes_{A} B\right) \cong\left(\lim _{\longrightarrow} M_{\lambda}\right) \otimes_{A} B \cong M \otimes_{A} B \rightarrow M
$$

of graded $B$-modules. Since $M_{\lambda} \otimes_{A} B$ equals $f^{*} M_{\lambda}$, it is in T; but T is closed under direct limits and quotients, so $M$ is in T. Thus $g_{*}$ is faithful.

The following consequence of the theorem slightly extends a result of Van den Bergh [13, Proposition 3.9.11].

Corollary 3.4. Let $\phi: A \rightarrow B$ be a homomorphism of graded rings such that $B$ becomes a finitely presented graded left A-module. Then $\phi$ induces an affine map $g: \operatorname{Proj}_{n c} B \rightarrow \operatorname{Proj}_{n c} A$.

Proof. If we apply $A / \mathfrak{m} \otimes_{A}$ - to a finite presentation of $B$ as a left $A$-module, we see that $B / \phi(\mathfrak{m}) B$ has finite dimension. Thus, as a right $A$-module $B / \phi(\mathfrak{m}) B$ is annihilated by $\mathfrak{m}^{n}$ for some $n \gg 0$. Equivalently, $B \phi(\mathfrak{m})^{n} \subset \phi(\mathfrak{m}) B$. Thus the hypotheses of the theorem are satisfied. It remains to show that $Z$ is empty.

Let $I$ denote the right annihilator in $B$ of $B / \phi(\mathfrak{m}) B$. We have already observed that $\phi(\mathfrak{m})^{n} \subset I$. Since $A / \mathfrak{m}^{n}$ is finite dimensional, so is $A / \mathfrak{m}^{n} \otimes_{A} B \cong B / \phi(\mathfrak{m})^{n} B$. Thus $B / I$ is finite dimensional. Hence the zero locus of $I$ in $\operatorname{Proj}_{n c} B$ is empty.

Remark. If, in Theorem 3.3, $B_{A}$ is finitely presented, then we have the useful technical fact that $g^{!} \pi=\pi^{\prime} f^{!}$. This follows from Lemma 3.1(3) once we show that $f^{!}$sends $\mathrm{Fdim} A$ to $\mathrm{F} \operatorname{dim} B$. Let $M=\underline{\lim } M_{\lambda}$ be a direct limit of finite dimensional $A$ modules. If $B$ is a finitely presented right $A$-module, then $\operatorname{Hom}_{\mathrm{Gr} A}(B,-)$ commutes with direct limits, so $\operatorname{Hom}_{\mathrm{Gr} A}\left(N, \lim _{\mathrm{M}} M_{\lambda}\right)=\varliminf_{\longrightarrow} \operatorname{Hom}_{\mathrm{Gr} A}\left(B, M_{\lambda}\right)$; this is a direct limit of finite dimensional $B$-modules, because $B_{A}$ is finitely generated. Hence $f^{!}(\operatorname{Fdim} A) \subset \operatorname{Fdim} B$.

\section{The Veronese MAPPing}

Throughout this section $A$ is a locally finite $\mathbb{N}$-graded $k$-algebra and $n$ is a positive integer. The $n^{\text {th }}$ Veronese subalgebra $A^{(n)}$ is defined by

$$
A_{i}^{(n)}:=A_{n i} .
$$

It is a classical result in algebraic geometry that if $A$ is a finitely generated commutative connected graded $k$-algebra generated in degree one, then Proj $A \cong \operatorname{Proj} A^{(n)}$. This isomorphism is implemented by the Veronese embedding.

Verevkin proved a non-commutative version of this result when $A$ is noetherian and generated in degree one [12, Theorem 4.4].

Theorem 4.1 and Proposition 4.8 show what happens when $A$ need not be commutative and need not be generated in degree one.

Theorem 4.1. Let $A$ be a left noetherian locally finite $\mathbb{N}$-graded $k$-algebra. Fix a positive integer $n$. There is a map $g: \operatorname{Proj}_{n c} A \rightarrow \operatorname{Proj}_{n c} A^{(n)}$. Furthermore, $g_{*}$ is exact and $g_{*} g^{*} \cong \mathrm{id}$. If $A$ is also right noetherian, then $g_{*}$ has a right adjoint $g^{!}$.

We will use the notation $X:=\operatorname{Proj}_{n c} A^{(n)}$ and $X^{\prime}:=\operatorname{Proj}_{n c} A$. 
We need two preliminary results before proving the theorem. First we explain how the functors defining the map $g: \operatorname{Proj}_{n c} A \rightarrow \operatorname{Proj}_{n c} A^{(n)}$ in the theorem are induced by functors between the categories $\operatorname{GrMod} A$ and $\operatorname{GrMod} A^{(n)}$.

If $L$ is a graded $A$-module, we define the graded $A^{(n)}$-module $L^{(n)}$ by

$$
L_{i}^{(n)}:=L_{n i} \text {. }
$$

The rule $L \mapsto L^{(n)}$ extends to give an exact functor

$$
f_{*}: \operatorname{GrMod} A \rightarrow \operatorname{GrMod} A^{(n)} .
$$

The functor $f_{*}$ is not faithful when $n \geq 2$, because $f_{*}((A / \mathfrak{m})(1))=0$.

Proposition 4.2. Let $A$ be a locally finite $\mathbb{N}$-graded $k$-algebra. Fix a positive integer $n$. Let $W$ and $W^{\prime}$ be the spaces with module categories

$$
\operatorname{Mod} W=\operatorname{GrMod} A^{(n)}
$$

and

$$
\operatorname{Mod} W^{\prime}=\operatorname{GrMod} A \text {. }
$$

Then there is a map $f: W^{\prime} \rightarrow W$ with direct image functor given by $f_{*} L=L^{(n)}$. Furthermore, $f_{*}$ has a right adjoint $f^{!}$.

Proof. It is clear that $f_{*}$ is an exact functor commuting with direct sums. By the graded version of Watt's Theorem, $f_{*} \cong-\bar{\otimes}_{A} M$, where

$$
M:=\bigoplus_{p \in \mathbb{Z}} A(p)^{(n)}
$$

with components ${ }_{p} M_{q}=\left(A(p)^{(n)}\right)_{q}=A(p)_{n q}$. The right action of $A^{(n)}$ on $M$ is given by right multiplication, and each $A(p)^{(n)}$ is a right $A^{(n)}$-submodule. The left action of $A$ is given by left multiplication, whereby $a \in A_{i}$ acts by sending $A(p)_{n q}$ to $A(p+i)_{n q}$.

Define $f^{*}: \operatorname{GrMod} A^{(n)} \rightarrow \operatorname{GrMod} A$ by $f^{*} N=N \otimes_{A^{(n)}} A$ with the usual right action of $A$, and grading given by

$$
\left(N \otimes_{A^{(n)}} A\right)_{s}=\sum_{n i+j=s} N_{i} \otimes A_{j} .
$$

It is not hard to show that $f^{*}$ is a left adjoint to $f_{*}$. Therefore $f^{*} \cong-\bar{\otimes}_{A^{(n)}} Q$, where

$$
Q=\bigoplus_{p \in \mathbb{Z}} f^{*}\left(A^{(n)}(p)\right) \cong \bigoplus_{p \in \mathbb{Z}} A(n p)
$$

multiplication $A^{(n)}(p) \otimes_{A^{(n)}} A \rightarrow A(n p)$ gives an isomorphism of graded right $A$ modules. Thus ${ }_{p} Q_{*} \cong A(n p)$ with its usual grading. One can verify directly that $f_{*} \cong \underline{\underline{\operatorname{Hom}}}_{A}(Q,-)$.

The right adjoint to $f_{*}$ is the functor $f^{!}=\underline{\underline{\operatorname{Hom}}}_{A^{(n)}}(M,-)$. If $N$ is a graded right $A^{(n)}$-module, then

$$
\left(f^{!} N\right)_{i}=\operatorname{Hom}_{\mathrm{Gr} A^{(n)}}\left({ }_{-i} M_{*}, N\right)=\operatorname{Hom}_{\mathrm{Gr} A^{(n)}}\left(A(-i)^{(n)}, N\right) .
$$

If $N$ is a graded $A^{(n)}$-module, then $f_{*} f^{*}(N)=N$, so $f_{*} f^{*}$ is naturally equivalent to $\operatorname{id}_{W}$. 
Let $\pi^{\prime}: \operatorname{GrMod} A \rightarrow$ Tails $A$ and $\pi: \operatorname{GrMod} A^{(n)} \rightarrow$ Tails $A^{(n)}$ be the quotient functors. To prove Theorem 4.1, we must find functors $g^{*}, g_{*}$, and $g^{!}$making the following diagrams commute:
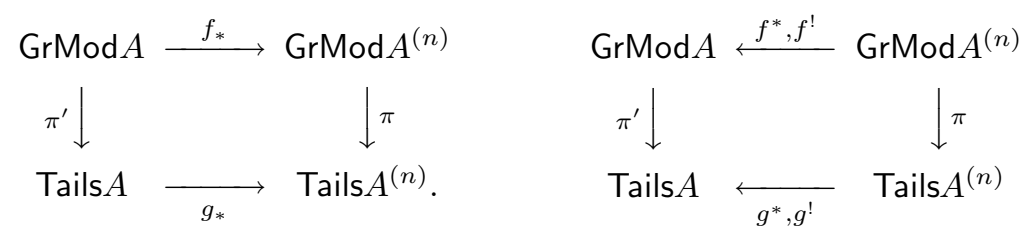

where $f^{*}, f_{*}$, and $f^{!}$, are the functors in the previous proposition.

Since $f_{*}$ sends Fdim $A$ to $\operatorname{Fdim} A^{(n)}$, there is a functor $g_{*}: \operatorname{Tails} A \rightarrow \operatorname{Tails} A^{(n)}$ such that $g_{*} \pi^{\prime}=\pi f_{*}$. Because $f_{*}$ is exact, $g_{*}$ is too [6].

To ensure that $f^{*}$ and $f^{!}$induce functors between the quotient categories, we must impose a noetherian hypothesis. Although there is no noetherian hypothesis in Proposition 4.2, in Theorem 4.1 it is assumed that $A$ is right noetherian. This hypothesis ensures that $f^{*}$ sends $\operatorname{Fdim} A^{(n)}$ to $\operatorname{Fdim} A$.

Recall that GrMod $A$ denotes the category of graded right $A$-modules.

Lemma 4.3. Let $A$ be a locally finite $\mathbb{N}$-graded $k$-algebra. Then:

(1) $f_{*}$ sends right noetherian $A$-modules to right noetherian $A^{(n)}$-modules;

(2) if $A$ is right noetherian so is $A^{(n)}$, and $A$ is a finitely generated right $A^{(n)}$. module.

(3) if $A$ is left noetherian, then $f^{*} \operatorname{sends} \operatorname{Fdim} A^{(n)}$ to $\operatorname{Fdim} A$;

(4) if $A$ is left noetherian, then there is a functor $g^{*}:$ Tails $A^{(n)} \rightarrow$ Tails $A$ such that $g^{*} \pi=\pi^{\prime} f^{*}$

(5) if $A$ is right noetherian, then $f^{!} \operatorname{sends} \operatorname{Fdim} A^{(n)}$ to $\operatorname{Fdim} A$;

(6) if $A$ is right noetherian, then there is a functor $g^{!}:$Tails $A^{(n)} \rightarrow$ Tails $A$ such that $g^{!} \pi=\pi^{\prime} f^{!}$;

Proof. (1) Let $M$ be a right noetherian graded $A$-module. If $N$ is a submodule of $M^{(n)}$, then $N=N A \cap M^{(n)}$. Hence any proper ascending chain of submodules in $M^{(n)}$ would give a proper ascending chain of submodules of $M$ by multiplying by $A$. Since $M$ contains no such chain, neither does $M^{(n)}$.

(2) Applying (1) to $M=A$ shows that $A^{(n)}$ is right noetherian.

Applying (1) to $M:=A \oplus A(1) \oplus \cdots \oplus A(n-1)$ gives the result, because $M^{(n)} \cong A$ as a right $A^{(n)}$-module.

(3) Because $A$ is left noetherian, the left module version of (2) implies that $A$ is a finitely generated left $A^{(n)}$-module. Hence if $N$ is a finite dimensional right $A^{(n)}$-module, $N \otimes_{A^{(n)}} A$ is a finite dimensional $A$-module. Thus $f^{*}$ sends finite dimensional right $A^{(n)}$-modules to finite dimensional right $A$-modules. Since $f^{*}$ is a left adjoint, it commutes with direct limits. The result follows.

(4) Because $A$ is left noetherian, we may invoke (3). The existence of such $g^{*}$ now exists by the universal property of the quotient functor $\pi$.

(5) First we show that it suffices to prove that $f^{!}$send finite dimensional $A^{(n)}$. modules to finite dimensional $A$-modules. To this end, let $N \in \operatorname{Fdim} A^{(n)}$ and write $N=\underline{\lim } N_{\lambda}$ as a direct limit of finite dimensional modules. Recall that 
$f^{!} N=\underline{\underline{\operatorname{Hom}}}_{A^{(n)}}(M, N)$, where

$$
M=\bigoplus_{p \in \mathbb{Z}} A(p)^{(n)} .
$$

By the noetherian hypothesis, $A(p)^{(n)}$ is a finitely generated, and hence finitely presented, right $A^{(n)}$-module, so $\operatorname{Hom}_{\mathrm{Gr} A^{(n)}}\left(A(p)^{(n)},-\right)$ commutes with direct limits. It follows that

$$
\begin{aligned}
f^{!}\left(\underline{\lim } N_{\lambda}\right) & =\bigoplus_{p} \operatorname{Hom}_{\mathrm{Gr}^{(n)}}\left(A(p)^{(n)}, \underline{\lim } N_{\lambda}\right) \\
& =\bigoplus_{p} \underline{\lim _{\longrightarrow}} \operatorname{Hom}_{\mathrm{Gr} A^{(n)}}\left(A(p)^{(n)}, N_{\lambda}\right) \\
& =\varliminf_{\lim } \bigoplus_{p} \operatorname{Hom}_{\mathrm{Gr}^{(n)}}\left(A(p)^{(n)}, N_{\lambda}\right) \\
& =\varliminf_{\lim } f^{!}\left(N_{\lambda}\right) .
\end{aligned}
$$

Hence, if each $f^{!}\left(N_{\lambda}\right)$ is finite dimensional, $f^{!} N$ is a direct limit of finite dimensional modules.

Now we show that $f^{!} N$ is finite dimensional when $N$ is a finite dimensional graded right $A^{(n)}$-module. It suffices to show that $\left(f^{!} N\right)_{-p}$, which is equal to $\operatorname{Hom}_{\mathrm{Gr} A^{(n)}}\left(A(p)^{(n)}, N\right)$, is zero for almost all $p$ and is finite dimensional for all $p$.

By the noetherian hypothesis, $A(p)^{(n)}$ is a finitely generated right $A^{(n)}$-module, so $\operatorname{Hom}_{\mathrm{Gr} A^{(n)}}\left(A(p)^{(n)}, N\right)$ has finite dimension.

We now show that $\operatorname{Hom}_{\mathrm{Gr} A^{(n)}}\left(A(p)^{(n)}, N\right)$ is zero if $|p|$ is sufficiently large. Fix $p$. For every integer $j$ we have

$$
A(p+n j)^{(n)} \cong A(p)^{(n)}(j)
$$

so

$$
\operatorname{Hom}_{\mathrm{Gr}^{(n)}}\left(A(p+n j)^{(n)}, N\right) \cong \operatorname{Hom}_{\mathrm{Gr} A^{(n)}}\left(A(p)^{(n)}, N(-j)\right) .
$$

Since $A(p)^{(n)}$ is finitely generated and $N$ is finite dimensional, when $|j|$ is sufficiently large $\operatorname{Hom}_{\mathrm{Gr} A^{(n)}}\left(A(p)^{(n)}, N(-j)\right)$ is zero. Hence $\operatorname{Hom}_{\mathrm{Gr} A^{(n)}}\left(A(p)^{(n)}, N\right)$ is zero for $|p|$ sufficiently large. This completes the proof that $f^{!}$sends finite dimensional modules to finite dimensional modules.

(6) follows from (5) in the same way that (4) follows fom (3).

Proof of Theorem 4.1. By Lemma 4.3 there are functors $g^{*}$ and $g_{*}$ between the categories $\operatorname{Mod} \operatorname{Proj}_{n c} A^{(n)}=\operatorname{Tails} A^{(n)}$ and $\operatorname{Mod}_{\operatorname{Proj}} A=$ Tails $A$ satisfying

$$
g^{*} \pi=\pi^{\prime} f^{*}, \quad g_{*} \pi^{\prime}=\pi f_{*}, \quad g^{!} \pi=\pi^{\prime} f^{!} .
$$

Applying Lemma 3.1 to $f^{*}$, we see that $g^{*}$ has $\pi f_{*} \omega^{\prime}$ as a right adjoint. But $\pi f_{*} \omega^{\prime}=g_{*} \pi^{\prime} \omega^{\prime}=g_{*}$, so $g_{*}$ is a right adjoint to $g^{*}$. We have already remarked that $g_{*}$ is exact because $f_{*}$ is. Furthermore,

$$
\mathrm{id} \cong \pi \omega \cong \pi f_{*} f^{*} \omega=g_{*} \pi^{\prime} f^{*} \omega=g_{*} g^{*} \pi \omega \cong g_{*} g^{*} .
$$

Since $f^{*} A^{(n)}=A, g^{*} \mathcal{O}_{\operatorname{Proj}_{n c} A^{(n)}}=\mathcal{O}_{\operatorname{Proj}_{n c} A}$.

Now suppose that $A$ is also right noetherian. Then $g_{*}$ has a right adjoint $g$ ! by Lemma 4.3 and $g^{\prime} \pi=\pi^{\prime} f^{!}$. This completes the proof of Theorem 4.1 
In the next result $\operatorname{Proj} A$ is the usual commutative scheme viewed as a noncommutative space with module category Qcoh(Proj $A)$.

Corollary 4.4. If $A$ is a finitely generated commutative connected graded $k$-algebra, there is a map $g: \operatorname{Proj}_{n c} A \rightarrow \operatorname{Proj} A$. Furthermore, $g_{*}$ has a right adjoint $g !$.

Proof. For some sufficiently large $n, A^{(n)}$ is generated in degree one; so

$$
\text { Tails } A^{(n)} \cong \text { Qcoh Proj } A^{(n)}=\text { Qcoh Proj } A \text {. }
$$

Hence $\operatorname{Proj}_{n c} A^{(n)} \cong \operatorname{Proj} A$. Therefore Theorem 4.1 gives the result.

Remarks. 1. Suppose that $A$ is both left and right noetherian, as in Theorem 4.1 Since $g_{*}$ has both a left and a right adjoint, it is exact; its right adjoint $g$ ! therefore preserves injectives. Hence there is a convergent spectral sequence

$$
\operatorname{Ext}_{\operatorname{Proj}_{n c} A}^{p}\left(M, R^{q} g^{!} N\right) \Rightarrow \operatorname{Ext}_{\operatorname{Proj}_{n c} A^{(n)}}^{p+q}\left(g_{*} M, N\right)
$$

for $M$ and $N$ modules over $\operatorname{Proj}_{n c} A$ and $\operatorname{Proj}_{n c} A^{(n)}$ respectively.

2. If $J$ is a two-sided ideal of $A$, then the natural map $A \rightarrow A / J$ induces an isomorphism $A^{(n)} / J^{(n)} \rightarrow(A / J)^{(n)}$, so there is a commutative diagram

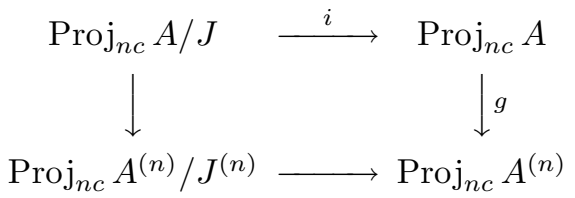

where the horizontal maps are the natural closed immersions.

If $A$ is prime, right noetherian, we define

$$
\text { Fract }_{g r} A:=\left\{a b^{-1} \mid a, b \in A \text { are homogeneous and } b \text { is regular }\right\} \text {. }
$$

Proposition 4.5. Let $A$ be a right noetherian, locally finite, $\mathbb{N}$-graded $k$-algebra. Suppose that $A$ is prime and Fract ${ }_{g r} A$ contains a copy of $A(n)$ for all $n \in \mathbb{Z}$. Then

(1) $\operatorname{Proj}_{n c} A$ and $\operatorname{Proj}_{n c} A^{(n)}$ are integral spaces in the sense of [10], and

(2) $g: \operatorname{Proj}_{n c} A \rightarrow \operatorname{Proj}_{n c} A^{(n)}$ is a birational isomorphism in the sense that it induces an isomorphism between the function fields.

Proof. That $\operatorname{Proj}_{n c} A$ is an integral space is proved in [10, Th. 4.5]. It is also shown there that the function field of $\operatorname{Proj}_{n c} A$ is isomorphic to $\left(\text { Fract }_{g r} A\right)_{0}$. It is clear that $\left(\text { Fract }_{g r} A^{(n)}\right)_{0} \subset\left(\text { Fract }_{g r} A\right)_{0}$, and the reverse inclusion follows from the observation that $a b^{-1}=a b^{n-1} b^{-n}$.

Remarks. 1. If $A$ is prime noetherian and has a regular element of degree $d$ for all $d \gg 0$, then Fract gr $A$ contains a copy of $A(n)$ for all $n \in \mathbb{Z}$, so the previous result applies.

2. If $z$ is a normal regular element, then the complement in $\operatorname{Proj}_{n c} A$ to the zero locus of $z$ is the open subspace $U:=\operatorname{Proj}_{n c} A\left[z^{-1}\right]$. Now $\operatorname{Mod} U$ is equivalent to $\operatorname{GrMod} A\left[z^{-1}\right]$. If $d$ is the smallest positive integer such that $A\left[z^{-1}\right]$ has a unit of degree $d$, then $U$ is an affine space with coordinate ring

$$
\left(\begin{array}{cccc}
R_{0} & R_{1} & \cdots & R_{d-1} \\
R_{-1} & R_{0} & \cdots & R_{d-2} \\
\vdots & & & \vdots \\
R_{-d+1} & R_{-d+2} & \cdots & R_{0}
\end{array}\right)
$$


where $R=A\left[z^{-1}\right]$. The reason for this is that $\bigoplus_{i=0}^{d-1} A\left[z^{-1}\right](i)$ is a generator in $\operatorname{GrMod} A\left[z^{-1}\right]$ and the tiled matrix ring is the endomorphism of this generator.

3. In the situation of Proposition 4.5, if $s$ and $t$ are homogeneous regular elements of relatively prime degrees in $A$, and $s t$ is normal, meaning that $s t A=A s t$, then $A\left[(s t)^{-1}\right]$ has a unit of degree one; so, if $U$ is the open complement to the zero locus of $s t$ in $\operatorname{Proj}_{n c} A$, then $U$ is the affine space with coordinate ring $A\left[(s t)^{-1}\right]_{0}$. This ring is equal to $A^{(n)}\left[(s t)^{-n}\right]_{0}$, so the open complement is isomorphic to the open complement to the zero locus of $(s t)^{n}$ in $\operatorname{Proj}_{n c} A^{(n)}$.

4. If Fract ${ }_{g r} A$ fails to contain a copy of every $A(n)$, the map $\operatorname{Proj}_{n c} A \rightarrow$ $\operatorname{Proj}_{n c} A^{(n)}$ need not be a birational isomorphism. For example, take $A=k[x]$ with $\operatorname{deg} x=2$.

Example 4.6. If $A$ is not generated in degree one, then $g_{*}$ need not be faithful.

Let $A=k[x, z]$ be the polynomial ring with $\operatorname{deg} x=1$ and $\operatorname{deg} z=n \geq 2$. The image under $\pi$ of $M=A /(x)$ is a simple module $\mathcal{O}_{p}$ in $\operatorname{Proj}_{n c} A$. We have $\mathcal{O}_{p}(1) \neq 0$, but $(M(1))^{(n)}=0$, so $g_{*}\left(\mathcal{O}_{p}(1)\right)=0$.

One might anticipate that $g: \operatorname{Proj}_{n c} A \rightarrow \operatorname{Proj}_{n c} A^{(n)}$ is an isomorphism on suitable open subspaces: in the previous example, $g$ restricts to an isomorphism from the complement to the zero locus of $x$ in $\operatorname{Proj}_{n c} A$ to the complement to the zero locus of $x$ in $\operatorname{Proj}_{n c} A^{(n)}$. We prove a general result of this type in Proposition 4.8. First we need a lemma.

For each integer $r$, define

$$
A^{(n)+r}:=\sum_{j \in \mathbb{Z}} A_{n j+r} .
$$

Obviously $A^{(n)+r} A^{(n)+s} \subset A^{(n)+r+s}$, so each $A^{(n)+r}$ is an $A^{(n)}-A^{(n)}$-bimodule, and these bimodules depend only on $r(\bmod n)$. Define

$$
I_{r}:=A^{(n)+r} A=\sum_{j \in \mathbb{Z}} A_{n j+r} A
$$

and

$$
I:=\bigcap_{r \in \mathbb{Z}} I_{r}=I_{1} \cap I_{2} \cap \cdots \cap I_{n} .
$$

Although $I_{r}$ is in general only a right ideal of $A, I_{r}^{(n)}$ is a two-sided ideal of $A^{(n)}$. Since $A_{q} I_{r} \subset I_{q+r}, I$ is a two-sided ideal of $A$.

Notice that $A^{(n)+r} A^{(n)-r}=I_{r}^{(n)}$.

Lemma 4.7. With the above notation, $I^{2 n} \subset I^{(n)} A$.

Proof. From the containment

$$
I^{2} \subset I_{r} I=A^{(n)+r} I \subset A^{(n)+r} I_{n-r}=A^{(n)+r} A^{(n)+n-r} A=I_{r}^{(n)} A,
$$

it follows that

$$
I^{2 n} \subset I_{1}^{(n)} I^{2 n-2} \subset I_{1}^{(n)} I_{2}^{(n)} I^{2 n-4} \subset \cdots \subset I_{1}^{(n)} \cdots I_{n}^{(n)} A .
$$

But this last term is contained in

$$
\left(I_{1}^{(n)} \cap \cdots \cap I_{n}^{(n)}\right) A=I^{(n)} A,
$$

which completes the proof. 
Proposition 4.8. With the hypotheses of Theorem 4.1, the map g restricts to an isomorphism $g: \operatorname{Proj}_{n c} A \backslash Z^{\prime} \rightarrow \operatorname{Proj}_{n c} A^{(n)} \backslash Z$, where $Z^{\prime}$ and $Z$ are the zero loci of $I$ and $I^{(n)}$ respectively.

Proof. Write $X^{\prime}=\operatorname{Proj}_{n c} A, X=\operatorname{Proj}_{n c} A^{(n)}, U^{\prime}=X^{\prime} \backslash Z^{\prime}$ and $U=X \backslash Z$. Write $\alpha: U \rightarrow X$ and $\beta: U^{\prime} \rightarrow X^{\prime}$ for the inclusions. We will use Lemma 3.1 to show that there is an isomorphism $h: U^{\prime} \rightarrow U$ such that the diagram

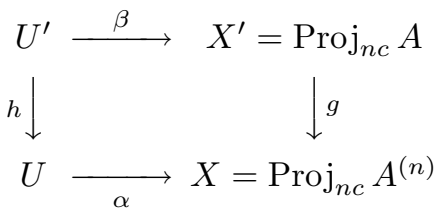

commutes.

Let $\mathrm{T}$ be the localizing subcategory of GrMod $A$ consisting of those modules $L$ such that every element of $L$ is killed by a suitably large power of $I$. Let $\mathrm{S}$ be the localizing subcategory of $\operatorname{GrMod} A^{(n)}$ consisting of those modules $N$ such that every element of $N$ is killed by a suitably large power of $I^{(n)}$. These two localizing subcategories contain all the finite dimensional modules. The spaces $U$ and $U^{\prime}$ are defined by

$$
\operatorname{Mod} U:=\left(\operatorname{GrMod} A^{(n)}\right) / \mathrm{S} \text { and } \operatorname{Mod} U^{\prime}:=(\operatorname{GrMod} A) / \mathrm{T} .
$$

Let $f_{*}$ and $f^{*}$ be the functors defined in Proposition 4.2. We will show that $f_{*}(\mathrm{~T}) \subset \mathrm{S}$ and $f^{*}(\mathrm{~S}) \subset \mathrm{T}$. The first of these inclusions is obvious: if every element of an $A$-module $L$ is annihilated by a power of $I$, then every element of $L^{(n)}$ is annihilated by a power of $I^{(n)}$. To show that $f^{*}(\mathrm{~S}) \subset \mathrm{T}$, it suffices to show that $f^{*}\left(A^{(n)} / I^{(n)}\right)$ belongs to T. But $f^{*}\left(A^{(n)} / I^{(n)}\right) \cong A / I^{(n)} A$, and by Lemma 4.7 $A / I^{(n)} A$ is annihilated by $I^{2 n}$ so belongs to $\mathrm{T}$.

We now use Lemma 3.1 in the context of the following diagram:

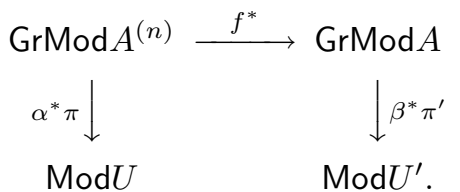

Because $f^{*}(\mathrm{~S}) \subset \mathrm{T}$, there exists a functor $h^{*}: \operatorname{Mod} U \rightarrow \operatorname{Mod} U^{\prime}$ such that $h^{*} \alpha^{*} \pi=$ $\beta^{*} \pi^{\prime} f^{*}$. Because $f_{*}$ is right adjoint to $f^{*}, h_{*}:=\alpha^{*} \pi f_{*} \omega^{\prime} \beta_{*}$ is right adjoint to $h^{*}$. Thus, $h^{*}$ and $h_{*}$ define a map $h: U^{\prime} \rightarrow U$. Since $g_{*} \pi^{\prime}=\pi f_{*}$, a computation gives $\alpha_{*} h_{*} \cong g_{*} \beta_{*}$. Therefore $\alpha h=g \beta$.

It remains to show that $h$ is an isomorphism.

The unit $\operatorname{id}_{\operatorname{GrMod} A^{(n)}} \rightarrow f_{*} f^{*}$ is an isomorphism because the natural map $L \rightarrow$ $\left(L \otimes_{A^{(n)}} A\right)^{(n)}$ is an isomorphism for all $L \in \operatorname{GrMod} A^{(n)}$. Because $f_{*}(\mathrm{~T}) \subset \mathrm{S}$, part (3) of Lemma 3.1 gives $h_{*} \beta^{*} \pi^{\prime} \cong \alpha^{*} \pi f_{*}$. Therefore,

$$
h_{*} h^{*} \cong h_{*} h^{*} \alpha^{*} \pi \omega \alpha_{*}=h_{*} \beta^{*} \pi^{\prime} f^{*} \omega \alpha_{*} \cong \alpha^{*} \pi f_{*} f^{*} \omega \alpha_{*} \cong \operatorname{id}_{U} .
$$

To show that the natural transformation $h^{*} h_{*} \rightarrow \mathrm{id}_{U^{\prime}}$ is an isomorphism, we first consider the natural transformation $f^{*} f_{*} \rightarrow \operatorname{id}_{\text {GrMod } A}$. For an $A$-module $M$ this is the multiplication map

$$
f^{*} f_{*} M=M^{(n)} \otimes_{A^{(n)}} A \rightarrow M .
$$

We claim that the kernel and cokernel of this map belong to $T$. 
Suppose that $\sum m_{i} \otimes a_{i} \in M^{(n)} \otimes_{A^{(n)}} A$ is in the kernel. Then $\sum_{i} m_{i} a_{i}=0$. By taking homogeneous components we can reduce to the case where each $a_{i}$ belongs to $A_{n-r}+A_{2 n-r}+\cdots$ for some $r \in\{1, \ldots, n\}$. Then, if $b \in A_{n j+r}$ for some $j$, then

$$
\left(\sum_{i} m_{i} \otimes a_{i}\right) b=\sum_{i} m_{i} \otimes a_{i} b=\sum_{i} m_{i} a_{i} b \otimes 1=0 .
$$

Thus $\left(\sum_{i} m_{i} \otimes a_{i}\right) I_{r}=0$. Hence the kernel is annihilated by $I$, so belongs to $\mathrm{T}$. The cokernel of $f^{*} f_{*} M \rightarrow M$ is $M / M^{(n)} A$. If $r \in\{1, \ldots, n\}$, then $M_{n j-r} I_{r} \subset M^{(n)} A$. Hence $I$ annihilates the cokernel.

Since the kernel and cokernel of $f^{*} f_{*} \rightarrow$ id belong to $\mathrm{T}$, there is an isomorphism $\beta^{*} \pi^{\prime} f^{*} f_{*} \rightarrow \beta^{*} \pi^{\prime}$. Hence

$$
h^{*} h_{*}=h^{*} \alpha^{*} \pi f_{*} \omega^{\prime} \beta_{*} \cong \beta^{*} \pi^{\prime} f^{*} f_{*} \omega^{\prime} \beta_{*} \cong \operatorname{id}_{U^{\prime}} .
$$

In Example 4.6, $I_{1}=(x)$ and $I_{2}=A$, so $I=(x)$, whence $Z^{\prime}$ is the zero locus of $x$. This explains why we need to remove the zero locus of $x$ to get the isomorphism.

If $A$ is generated in degree one, then $I_{r}=A_{\geq r}$ for $r \in\{0,1, \ldots, n-1\}$, so $A / I_{r} \in \mathrm{F} \operatorname{dim} A$, whence $A / I \in \mathrm{F} \operatorname{dim} A$. It follows that $Z$ and $Z^{\prime}$ are empty, and therefore $U=X$ and $U^{\prime}=X^{\prime}$. We therefore recover Verevkin's result $X \cong X^{\prime}$ when $A$ is generated in degree one over $A_{0}$.

Example 4.9. Let $A$ be a weighted polynomial ring. That is, $A=k\left[x_{0}, \ldots, x_{n}\right]$, where $\operatorname{deg} x_{i}=q_{i} \geq 1$. Write $Q=\left(q_{0}, \ldots, q_{n}\right)$. Then $\mathbb{P}_{Q}^{n}:=\operatorname{Proj} A$ is called a weighted projective space. It is isomorphic to the quotient variety $\mathbb{P}^{n} / \mu_{Q}$, where $\mu_{Q}=\mu_{q_{0}} \times \cdots \times \mu_{q_{n}}$. There is a sufficiently divisible integer $d$ such that $A^{(d)}$ is generated in degree one. Hence

$$
\mathbb{P}_{Q}^{n}=\operatorname{Proj} A=\operatorname{Proj} A^{(d)} \cong \operatorname{Proj}_{n c} A^{(d)} .
$$

By Theorem 4.1, there is a map

$$
g: \operatorname{Proj}_{n c} A \rightarrow \operatorname{Proj}_{n c} A^{(d)} \cong \mathbb{P}_{Q}^{n}
$$

This is an isomorphism on an open subspace by Proposition 4.8 Since $A$ has global homological dimension $n+1, \operatorname{Proj}_{n c} A$ has global homological dimension $n$. We therefore think of $\operatorname{Proj}_{n c} A$ as a smooth space of dimension $n$ and the map $g$ as a non-commutative resolution of $\mathbb{P}_{Q}^{n}$. Let $X \subset \mathbb{P}_{Q}^{n}$ be the closed subscheme cut out by an ideal $J$ in $A$. Then there is a commutative diagram

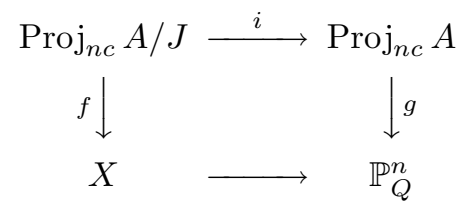

in which $f$ is a birational isomorphism and $i$ is a closed immersion. It can happen that $\operatorname{Proj}_{n c} A / J$ is smooth even when $X$ is singular. Thus $\operatorname{Proj}_{n c} A$ is a "noncommutative resolution" of $X$. An interesting case to examine in some detail is that where $X$ is an orbifold of a Calabi-Yau three-fold.

If $A=k[x]$ with $\operatorname{deg} x=2$, and $n=2$, then $\operatorname{Proj}_{n c} A \cong \operatorname{Spec} k \times k$ and $\operatorname{Proj} A \cong$ Spec $k$. Furthermore, $Z^{\prime}=X^{\prime}$ and $Z=X$. This is a special case of the next result, the truth of which was suggested by Darin Stephenson. 
Proposition 4.10. Let $A$ be a locally finite $\mathbb{N}$-graded $k$-algebra such that $A_{i}=0$ whenever $i \not \equiv 0(\bmod n)$. Then $\operatorname{Proj}_{n c} A$ is isomorphic to the disjoint union of $n$ copies of $\operatorname{Proj}_{n c} A^{(n)}$.

Proof. Let $p_{r}: \operatorname{GrMod} A \rightarrow \operatorname{GrMod} A$ be the functor defined by

$$
p_{r}(M)=\bigoplus_{i \in \mathbb{Z}} M_{r+i n}
$$

on objects, and $p_{r}(\theta)=\left.\theta\right|_{p_{r}(M)}$ whenever $\theta \in \operatorname{Hom}_{\mathrm{Gr} A}(M, N)$. The hypothesis on $A$ ensures that each $p_{r}(M)$ is a graded $A$-submodule of $M$, so $p_{r}$ is indeed a functor from $\operatorname{GrMod} A$ to itself. It is clear that $\operatorname{id}_{\mathrm{GrMod} A}=p_{0} \oplus \cdots \oplus p_{n-1}$, where this direct sum is taken in the abelian category of $k$-linear functors from $\operatorname{GrMod} A$ to itself; essentially, this is the observation that $M=p_{0}(M) \oplus \cdots \oplus p_{n-1}(M)$, and that any map $\theta: M \rightarrow N$ of graded $A$-modules respects this decomposition. Furthermore, each $p_{r}$ is idempotent and the $p_{r}$ s are mutually orthogonal. It follows from this that there is a decomposition of $\operatorname{GrMod} A$ as a product of categories, each component being the full subcategory on which $p_{r}$ is the identity.

It is clear that the shift functor (1) cyclicly permutes these subcategories, so they are all equivalent to one another and $(n)$ is an autoequivalence of each component. However, any one of these categories together with its autoequivalence $(n)$ is equivalent to $\operatorname{GrMod} A^{(n)}$ with its autoequivalence (1). Thus $\operatorname{GrMod} A$ is equivalent to the product of $n$ copies of $\operatorname{GrMod} A^{(n)}$.

Finally, this decomposition descends to the Tails categories.

\section{An Ore extension and an example}

The morphism

$$
\begin{aligned}
p: \mathbb{P}^{n} \backslash\{(0, \ldots, 0,1)\} & \rightarrow \mathbb{P}^{n-1}, \\
\left(\alpha_{0}, \ldots, \alpha_{n}\right) & \mapsto\left(\alpha_{0}, \ldots, \alpha_{n-1}\right),
\end{aligned}
$$

is called the projection with center $(0, \ldots, 0,1)$. This section examines a noncommutative analogue of this basic operation.

Consider a connected graded $k$-algebra $R$ and a connected graded Ore extension

$$
S=R[t ; \sigma, \delta]
$$

with respect to a graded automorphism $\sigma$ and a graded $\sigma$-derivation $\delta$ of degree $n \geq 1$. Thus $S=\bigoplus_{n=0}^{\infty} R t^{n}$ and $t r=r^{\sigma} t+\delta(r)$ for all $r \in R$. Since $\delta\left(R_{i}\right) \subset R_{i+n}$ for all $i$, by setting $\operatorname{deg} t=n, S$ becomes a connected graded algebra.

One expects that the inclusion map $R \rightarrow S$ induces a map $\operatorname{Proj}_{n c} S \rightarrow \operatorname{Proj}_{n c} R$. Indeed, the projection map above can be obtained as a special case of this.

Let $\mathfrak{m}$ denote the augmentation ideal of $R$. Since $\delta(\mathfrak{m}) \subset \mathfrak{m}, \mathfrak{m} S$ is a two-sided ideal of $S$. Furthermore, $S / \mathfrak{m} S \cong k[t]$ as graded rings.

Proposition 5.1. With the above notation, let $Z$ denote the zero locus of $\mathfrak{m} S$ in $\operatorname{Proj}_{n c} S$.

(1) $Z \cong \operatorname{Spec} k^{\times n}$.

(2) There is an affine map $g: \operatorname{Proj}_{n c} S \backslash Z \rightarrow \operatorname{Proj}_{n c} R$.

Proof. (2) The existence of $g$ is a special case of Theorem 3.3. That theorem applies because $S \mathfrak{m} \subset \mathfrak{m} S$. 
(1) The quotient $\operatorname{ring} S / \mathfrak{m} S$ is isomorphic to the polynomial ring $k[t]$ with $\operatorname{deg} t=$ $n$, so this follows from Proposition 4.10,

We think of $\operatorname{Proj}_{n c} S$ as a "cone over $\operatorname{Proj}_{n c} R$ with vertex $Z$ ". It would be interesting to describe the "fibers" of the map $g$.

When $\operatorname{deg} t>1$, the Ore extension $S=R[t ; \sigma, \delta]$ is not generated by its elements of degree one. This sometimes causes technical problems; however, if $R$ is generated in degree one, then the $n^{\text {th }}$ Veronese $S^{(n)}$ is generated in degree one. We can then combine Theorems 3.3 and 4.1 to analyze the space with homogeneous coordinate $\operatorname{ring} S$ as follows.

Proposition 5.2. The inclusion of the $n$-Veronese subalgebras of $S$ and $S / \mathfrak{m} S$ gives a commutative diagram of rings and an induced commutative diagram of spaces as in the following diagrams:

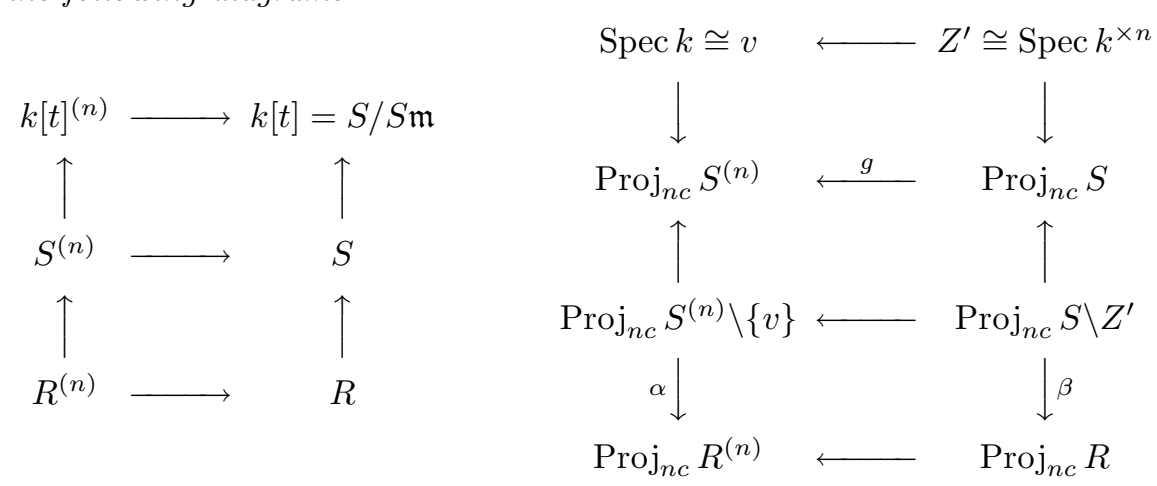

An application. In [11, a family of three-dimensional Artin-Schelter regular algebras $A$ is constructed and studied. Although the algebraic properties of $A$ are quite well understood, our understanding of the corresponding geometric object $\operatorname{Proj}_{n c} A$ is rudimentary. The algebras are of the form $A=R[t ; \sigma, \delta]$ with $\operatorname{deg} t=n$ and $R$ a two-dimensional Artin-Schelter regular algebra generated in degree one. It is well-known that $R$ and its Veronese subalgebras are (not necessarily commutative) homogeneous coordinate rings of $\mathbb{P}^{1}$. By Proposition 5.2, there is a commutative diagram of spaces and maps

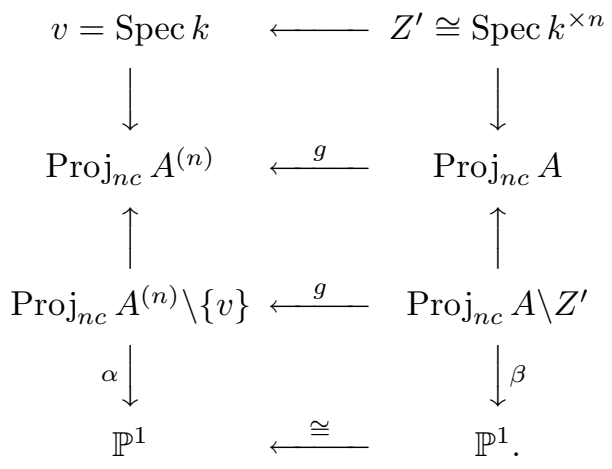

\section{ACKNOWLEDGEMEnts}

This work was stimulated by Darin Stephenson's paper [11]. I would like to thank him for explaining his results, and also suggesting that Proposition 4.10 should be true. The final paragraph of this paper applies our results to Stephenson's algebras. 
I thank James Zhang for bringing [3] to my attention. I thank the referee for suggesting several improvements to an earlier version of this paper.

\section{REFERENCES}

[1] M. Artin and J.J. Zhang, Non-commutative Projective Schemes, Adv. Math., 109 (1994), 228-287. MR 96a:14004

[2] V. Baranovsky, V. Ginzburg, and A. Kuznetzov, Quiver varieties and a noncommutative $\mathbb{P}^{2}$, Compositio Math., 134 (2002) 283-318.

[3] A. del Rio, Graded rings and equivalences of categories, Comm. Alg., 19 (1991) 997-1012. MR 92d:16011

[4] M. Beltrametti and L. Robbiano, Introduction to the theory of weighted projective spaces, Expositiones Math., 4 (1986) 111-162. MR 88h:14052

[5] I. Dolgachev, Weighted projective varieties, in Group actions and vector fields, pp. 34-71, Lecture Notes in Math. 956, Springer-Verlag, 1982. MR 85g:14060

[6] P. Gabriel, Des Catégories Abéliennes, Bull. Soc. Math. Fr., 90 (1962) 323-448. MR 38:1144

[7] A. Kapustin, A. Kuznetzov, and D. Orlov, Noncommutative Instantons and Twistor Transform, Comm. Math. Phys., 221 (2001) 385-432 MR 2003f:58017

[8] A.L. Rosenberg, Non-commutative algebraic geometry and representations of quantized algebras, Math. and Its Appl., Vol. 330, Kluwer Academic Publishers, 1995. MR 97b:14004

[9] S.P. Smith, Subspaces of non-commutative spaces, Trans. Amer. Math. Soc., 354 (2002) 2131-2171. MR 2003f: 14002

[10] S.P. Smith, Integral non-commutative spaces, J. Algebra, 246 (2001) 793-810. MR 2003d:16037

[11] D. Stephenson, Quantum planes of weight $(1,1, n)$, J. Algebra, 225 (2000) 70-92. Corrigendum, J. Algebra, 234 (2000) 277-278. MR 2001a:16044

[12] A.B. Verevkin, On a non-commutative analogue of the category of coherent sheaves on a projective scheme, Amer. Math. Soc. Transl. (2) 151 (1992) 41-53. MR 93j:14002

[13] M. Van den Bergh, Blowing up of a non-commutative smooth surface, Mem. Amer. Math. Soc., 154 (2001) no. 734. MR 2002k:16057

Department of Mathematics, Box 354350, University of Washington, Seattle, WashINGTON 98195

E-mail address: smith@math.washington.edu 